\title{
Larger surface area can reduce nitinol corrosion resistance
}

\author{
Grazziela M. Sena $\mathbb{D}^{1,2}$, Shiril Sivan $\mathbb{D}^{1}$, Jason D. Weaver $\mathbb{D}^{1}$ and Matthew Di Prima $\mathbb{D}^{1 凶}$
}

Surface area has long been thought to influence the corrosion behavior of medical devices although this phenomenon has not been systematically investigated. Because many nitinol implants are offered in a range of sizes, understanding the influence of device surface area on corrosion susceptibility is key to ensuring nitinol devices are safe. This study evaluates the corrosion susceptibility of amber oxide, mechanically polished and chemically etched nitinol wires with surface areas ranging from 0.05 to $10.0 \mathrm{~cm}^{2}$ using a standard test method, ASTM F2129. The corrosion testing shows that as the surface area increased the breakdown potential decreased. Results from the amber oxide and chemically etched samples show a plateauing of breakdown potential at larger surface areas, which suggests that there may be a minimum surface area threshold that could predict the performance of larger devices. Additional testing on electropolished wire and modified laser-cut stents shows a similar decrease in breakdown potential with increased surface area thus corroborating the trend observed with nitinol wires. Overall, surface area was found to affect corrosion susceptibility as measured per ASTM F2129 for a range of nitinol samples.

npj Materials Degradation (2020)4:23; https://doi.org/10.1038/s41529-020-00128-3

\section{INTRODUCTION}

As metallic implants are susceptible to corrosion after implantation, corrosion resistance testing is an important preclinical assessment which helps ensure long-term implant durability in the in vivo environment ${ }^{1-5}$. While there are multiple types of corrosion that can occur in medical devices such as galvanic, crevice, fretting, pitting, and metal ion release, this study will focus on pitting corrosion as determined by the standard test method, ASTM F2129 "Standard Test Method for Conducting Cyclic Potentiodynamic Polarization Measurements to Determine the Corrosion Susceptibility of Small Implant Devices" ${ }^{\prime \prime}$ that is well defined and is an FDA recognized consensus standard. Pitting corrosion results from a failure of the material's protective passive film, which leads to a localized breakdown at isolated sites ${ }^{7}$. The breakdown potential, $E_{\mathrm{b}}$, is commonly used to assess corrosion resistance with higher $E_{\mathrm{b}}$ values suggesting increased resistance to corrosion ${ }^{8}$. Corrosion can have serious consequences to patients with implanted devices as metal ion release during corrosion can cause adverse health effects and corrosion byproducts can be transported throughout the body through blood flow. Nickel has been shown to cause metal ion toxicity, formation of pseudotumors and kidney failure at high enough doses $^{9}$ and there is no known safe threshold to prevent a hypersensitivity response. While corrosion can affect the biocompatibility assessment ${ }^{10,11}$, biocompatibility is multifactorial with extensive and well established test standards to support it ${ }^{12,13}$. Specific biocompatibility concerns are not addressed in this manuscript. Corrosion on an implant could also lead to structural integrity problems as corrosion pits have been observed to initiate fatigue cracks in vitro ${ }^{14}$.

Nitinol is an example of a passive metal alloy that has been widely used in the biomedical field due to its unique properties including shape memory and psuedoelasticity ${ }^{1}$. While nitinol has been most commonly used in cardiovascular devices like stents, endovascular grafts, inferior vena cava filters, heart valves, etc., it is now being utilized in other areas, including respiratory, neurological, spinal, and orthopedic. As experience with nitinol has grown over the years and as nitinol devices are being used in different locations within the body, the importance of surface finish to its corrosion resistance has become recognized throughout the medical device community ${ }^{15,16}$. Highlighting the importance of surface finish to corrosion susceptibility, Sullivan et al. ${ }^{17}$ evaluated nitinol stents made with the same base material, but with five different surface finishes. The authors found that the breakdown potential varied from an average of $-117 \mathrm{mV}$ for an oxidized tubing finish to no breakdown observed up to $1000 \mathrm{mV}$ on stents with an electropolished (EP) finish.

ASTM F2129 details a number of experimental parameters (test temperature of $37 \pm 1{ }^{\circ} \mathrm{C}$, deaeration of the test solution with nitrogen gas, measurement of the open circuit potential of the test specimen for $1 \mathrm{~h}$ prior to initiating the potentiodynamic portion of the test, scan rate of the potentiodynamic portion of the test, etc.) to ensure repeatability and reproducibility among laboratories ${ }^{6}$. Multiple studies have investigated the effect of these parameters on test results. Increasing immersion time in phosphate-buffered saline (PBS), for example, has been found to shift the rest potential, $E_{r}$, to higher values with the amount of the shift being modulated by the exposure time and nitinol surface finish ${ }^{5,18-20}$. These same studies similarly found increases in $E_{\mathrm{b}}$ with longer immersion time which depended again on surface finish and whether breakdown was observed after the shorter immersion time. Previous research in our laboratory has additionally evaluated whether damage to the oxide layer through fatigue or fretting prior to testing could affect corrosion susceptibility ${ }^{19,21}$. Both studies found that such damage did not affect the observed breakdown potential of nitinol with differing surface finishes per ASTM F2129 which may have been due to repassivation of damaged regions. ASTM F2129 recommends PBS to be used as the standard test solution, although other solutions are allowed, and several formulations are provided in Appendix X2 of the document. Studies have examined the use of bovine blood, simulated gastric fluid, and PBS containing amino acids as a test solution and found that the test solution, in addition to the

'Division of Applied Mechanics, Office of Science and Engineering Laboratories, Center for Devices and Radiological Health, U.S. Food and Drug Administration, Silver Spring, MD 20993, USA. ${ }^{2}$ Oak Ridge Institute for Science and Education, Oak Ridge, TN, USA. ${ }^{\circledR}$ email: Matthew.Diprima@fda.hhs.gov 
immersion time and surface area, can all affect the observed $E_{\mathrm{r}}$ and $E_{\mathrm{b}}$ values ${ }^{22-24}$.

Although many metallic implants are available in several sizes to accommodate varying patient anatomies, ASTM F2129 does not currently provide recommendations on what device size to test except to say that the upper limit on specimen size may be limited by the current delivery capability of the test apparatus. For a given device and assuming uniform processing, a larger surface area may increase the likelihood that a flaw exists that would initiate pitting. This hypothesis is based off of a previously published model on the corrosion performance of stents which combined the probability of the stent pitting with the probability the breakdown potential would be below the acceptance criteria ${ }^{20}$. The statistical model for if a stent would pit was based off the exposed surface area and the number of occurrences per unit surface area. While the overall model matched the data in the study, it was limited to a single stent size and did not assess the influence of device size on the corrosion performance. Although the implant size selected for an individual patient depends primarily on anatomic and mechanical considerations, the question of how specimen surface area might affect corrosion susceptibility and which implant size to select for pre-clinical testing remains open. To that end, with this study, we attempt to characterize how surface area affects corrosion susceptibility per ASTM F2129 as well as the interplay of surface area and nitinol surface finish.

\section{RESULTS}

\section{Corrosion tests}

Figure 1 shows a plot of potential on $y$-axis and surface area on the $x$-axis of the average breakdown potentials of amber oxide (AO), mechanically polished (MP), chemically etched (CE), and EP wires as well as laser-cut stents. Statistical analysis (Table 1) showed that for AO, MP, and CE samples, there is a statistically significant difference $(p<0.05)$ in $E_{\mathrm{b}}$ between the smaller surface areas $\left(0.05-2.00 \mathrm{~cm}^{2}\right)$ and larger surface areas $\left(5.00-10.0 \mathrm{~cm}^{2}\right)$.

Figure 2 shows a bar graph of the percentage of the number of samples that experienced a breakdown at each surface area. It is worth noting that $\mathrm{AO}, \mathrm{MP}$, and $\mathrm{CE}$ all have a samples size of $N=10$ at each surface area, while EP wires and the stents were only tested at two surface areas with a sample size of $N=6$. The difference in the sample size is the reason for the representation in percentage instead of the actual sample count. The stent with the larger surface area was labeled as long stent, while the smaller one was labeled as short stent.

Figure 3 shows the distribution of breakdown potentials. This bar graph shows how many samples exhibited pitting corrosion at each potential. AO samples show a peak at about $600 \mathrm{mV}$, while for CE and MP samples are more widely distributed.

The average breakdown potential in Table 2 is tabulated in two categories: "w/NB" and "w/o NB" where NB stands for No Breakdown. Thus, the "w/NB" category average includes all specimens (specimens which reached the vertex potential without exhibiting breakdown were scored at $1000 \mathrm{mV}$ ). The "w/o NB" category average excludes specimens which reached the vertex potential without exhibiting breakdown. As previously stated, samples that did not breakdown are assumed to have a breakdown potential of $1000 \mathrm{mV}$.

\section{Poisson distribution}

Figures 4-6 show the plots of the probability of a certain number of pit occurrence of $A O, C E$, and MP at selected surface areas. The curve is the Poisson model while the red markers represent the actual number of counted pits. The $\lambda$ values were calculated based on the rate of pit occurrence. In the case of $\mathrm{AO}$, for example, the average number of pits at $10 \mathrm{~cm}^{2}$ is 89.90 pits. This gives an

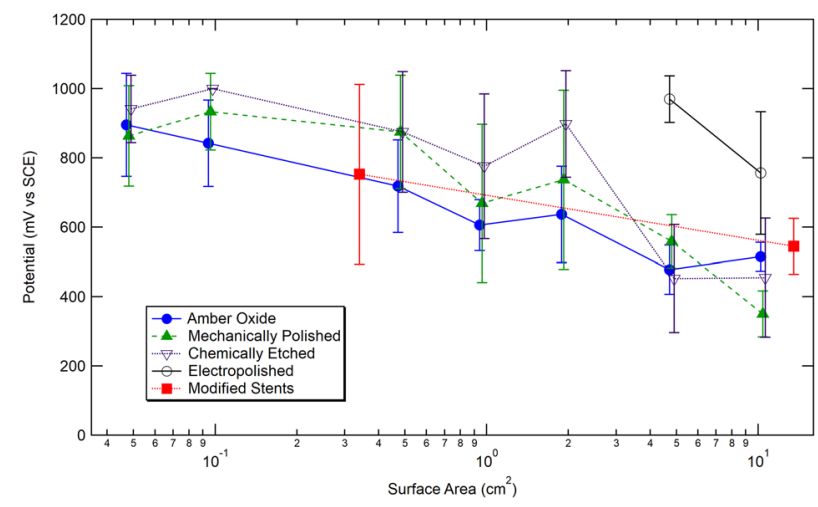

Fig. 1 Average breakdown potential vs. surface area. Plot of the average breakdown potential of $A O, M P, C E$, and $E P$ wires and modified stents at all surface areas tested. The vertex potential of $1000 \mathrm{mV}$ was used for samples that did not breakdown. Error bars represent \pm 1 standard deviation.

\begin{tabular}{|c|c|c|c|c|c|c|}
\hline Surface area $\left(\mathrm{cm}^{2}\right)$ & 0.10 & 0.50 & 1.00 & 2.00 & 5.00 & 10.00 \\
\hline \multicolumn{7}{|l|}{ a. Amber oxide } \\
\hline 0.05 & 0.159 & $0.003^{*}$ & $0.000^{*}$ & $0.004^{*}$ & $0.000^{*}$ & $0.000^{*}$ \\
\hline 0.10 & & 0.055 & $0.000^{*}$ & $0.017^{*}$ & $0.000^{*}$ & $0.000^{*}$ \\
\hline 0.50 & & & 0.031 & 0.401 & $0.000^{*}$ & $0.000^{*}$ \\
\hline 1.00 & & & & 0.536 & $0.003^{*}$ & $0.001 *$ \\
\hline 2.00 & & & & & $0.003^{*}$ & $0.002^{*}$ \\
\hline 5.00 & & & & & & 0.337 \\
\hline \multicolumn{7}{|c|}{ b. Mechanically polished } \\
\hline 0.05 & 0.212 & 0.596 & 0.122 & 0.566 & $0.000^{*}$ & $0.000^{*}$ \\
\hline 0.10 & & 0.583 & 0.01 & 0.11 & $0.000^{*}$ & $0.000^{*}$ \\
\hline 0.50 & & & 0.046 & 0.308 & $0.000^{*}$ & $0.000 *$ \\
\hline 1.00 & & & & 0.452 & 0.136 & $0.000^{*}$ \\
\hline 2.00 & & & & & $0.003^{*}$ & $0.000^{*}$ \\
\hline 5.00 & & & & & & $0.000^{*}$ \\
\hline \multicolumn{7}{|l|}{ c. Chemically etched } \\
\hline 0.05 & 0.067 & 0.555 & $0.048^{*}$ & 0.425 & $0.000^{*}$ & $0.000 *$ \\
\hline 0.10 & & $0.029^{*}$ & $0.001^{*}$ & $0.011^{*}$ & $0.000^{*}$ & $0.000 *$ \\
\hline 0.50 & & & 0.193 & 0.809 & $0.000^{*}$ & $0.000^{*}$ \\
\hline 1.00 & & & & & $0.001^{*}$ & $0.000^{*}$ \\
\hline 2.00 & & & & & $0.000^{*}$ & $0.000^{*}$ \\
\hline 5.00 & & & & & & 0.947 \\
\hline
\end{tabular}

average of $8.99 \mathrm{pits} / \mathrm{cm}^{2}$. To get the $\lambda$ values of the smaller surface areas, the average number of pits per surface area was used. The average number of pits per $1 \mathrm{~cm}^{2}$ of surface area for CE is 3.84 and $14.74 \mathrm{pits} / \mathrm{cm}^{2}$ for MP.

Table 3 shows the chi-square analysis between the Poisson model and observed number of pits on $\mathrm{AO}, \mathrm{MP}$, and CE samples at different surface areas. Chi-square analysis usually requires a sample size greater than 20 , since this study has a sample size of 10 , the calculated chi-square values had to be divided by the William's correction factor. This new value was then compared to the critical value at $a=0.05$ obtained from the chi-square table. 


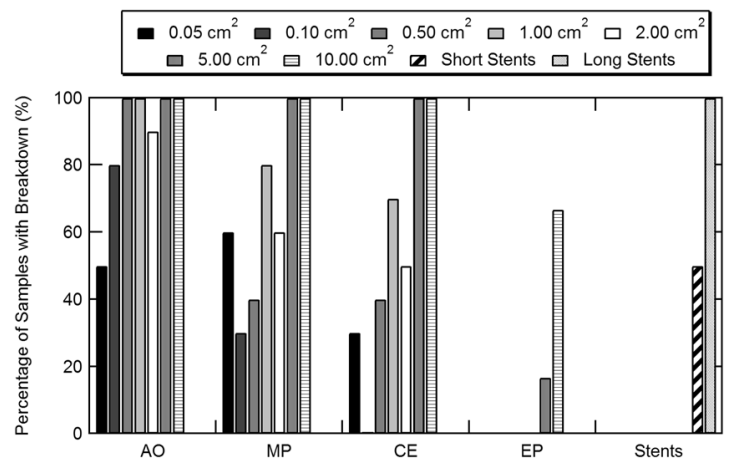

Fig. 2 Percent of Samples with Breakdown. Percentage of AO, MP, $\mathrm{CE}$, and EP wires and modified stent samples with breakdown at all surface areas tested. Note that only two different surface areas were tested for both EP wires and stents.

The Poisson model was deemed acceptable when the chi-square value with William's correction is less than the critical value.

\section{DISCUSSION}

This work set out to study the effect of surface area on pitting corrosion susceptibility. To account for differences in surface finish, three surface finishes of nitinol ( $A O, C E$, and MP) had their pitting corrosion susceptibility assessed from 0.05 to $10 \mathrm{~cm}^{2}$, and a fourth surface finish (EP) assessed for pitting corrosion susceptibility at 5 and $10 \mathrm{~cm}^{2}$. To account for geometry differences between straight wire and the complex shape of medical devices, as well as any differences between nitinol wire and laser cut nitinol tubing, two different lengths of nitinol stents with a thermally grown oxide were also assessed for pitting corrosion susceptibility. While this study did not investigate every metal, surface finish, or design used in medical devices, it did investigate a range of surface areas that extends above and below the average surface area of cardiovascular devices ${ }^{17,25}$ and materials whose breakdown potentials also match the mean breakdown potential of materials used in cardiovascular devices ${ }^{26}$.

As shown in Fig. 1, surface area had an observable effect on the pitting corrosion susceptibility on all surface finishes of nitinol. While this trend was expected, it was illuminating to observe how the trends differed across the surface areas. The different surfaces had differing critical surface areas above which corrosion was likely to occur. For $A O$ and $C E$ samples, the average breakdown potentials appeared to plateau to a minimum value at surface areas greater than $5 \mathrm{~cm}^{2}$; however, this result may not be generalizable to all implants made with these surface finishes. No such plateau was found in MP wires tested. Plateauing in the average breakdown potential was also evident in the statistical analysis shown in Table 1. The breakdown potentials observed at smaller surface areas were compared with the largest surface area. No statistical difference was found for $A O$ and CE samples between surface areas of 5 and $10 \mathrm{~cm}^{2}$. Interestingly, when comparing how many specimens experienced electrochemical breakdown (Fig. 2), the CE and MP surface finishes had similar numbers across the differing surface areas, while the AO surface finish experienced electrochemical breakdown more readily than the other two surface finishes. The EP surface finish experienced far fewer electrochemical breakdowns at 5 and $10 \mathrm{~cm}^{2}$ compared to the other surface finishes which is why smaller surface areas of EP nitinol wires were not investigated in this study. This also demonstrates the importance of surface finish on what the critical surface area is for a material to be susceptible to pitting corrosion.

Returning to the mechanics of pitting corrosion, pitting corrosion is expected to occur at a flaw or weak spot on the
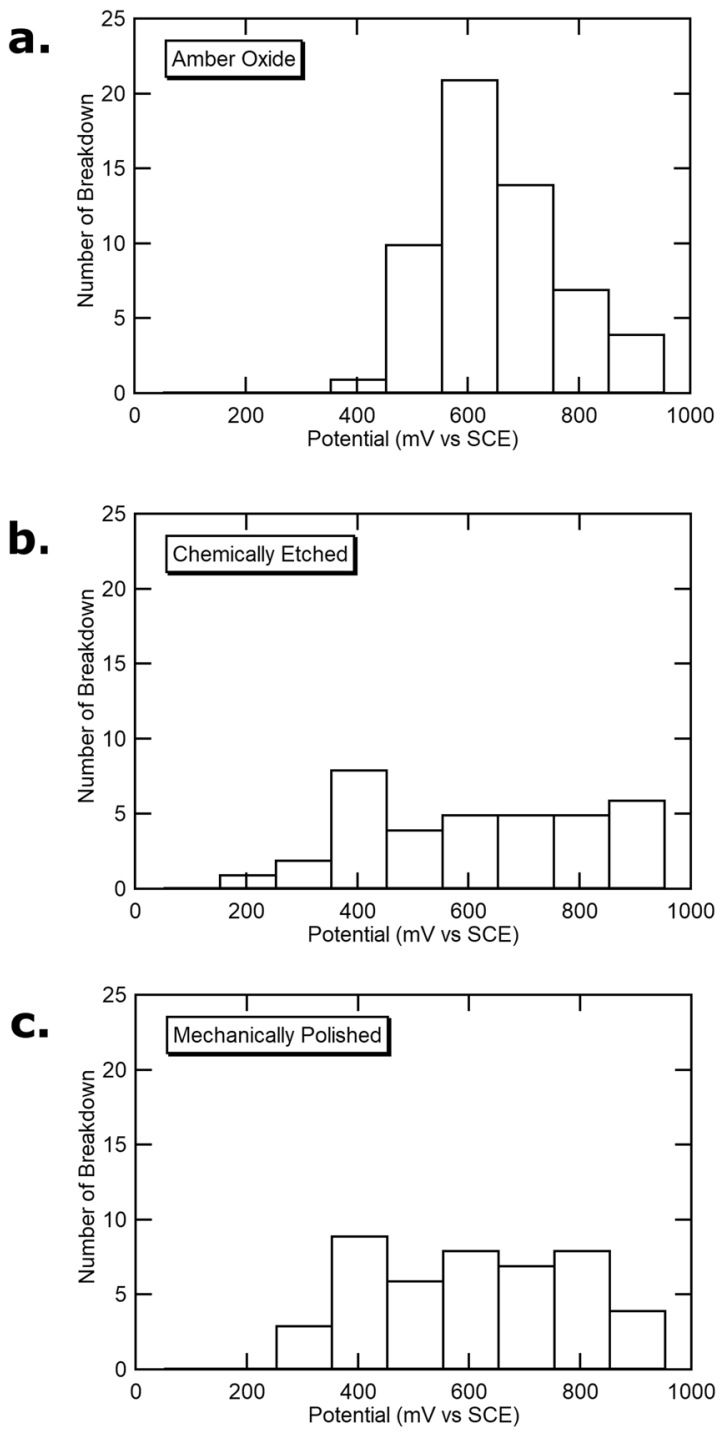

Fig. 3 Distribution of breakdown potential. Bar graphs showing the distribution of breakdown potentials of $\mathbf{a} \mathrm{AO}, \mathbf{b} \mathrm{CE}$, and $\mathbf{c} \mathrm{MP}$ wires over all surface areas tested.

oxide of the metal surface. This theory can explain Fig. 2 as each surface finish will result in a different distribution of surface flaws/ defects in the protective titanium oxide layer surrounding the nitinol wire. While there is some variability observed in Fig. 2, the general trend shows an increasing number of samples experiencing electrochemical breakdown with increasing surface area. To assess the statistical variability, these results were plotted against the Poisson distribution calculated based on the number of pits per surface area of the largest samples (Figs 4-6). Based on the chi-square test (Table 3), these figures show a generally good fit at lower surface areas with more divergence at larger surface areas. While this may be a limitation of using a sample size of 10 for each surface area, the practical utility of this approach is to assess what the critical surface area is for each material surface finish when pitting corrosion is likely to occur (when the probability of one or more pits becomes likely).

While the Poisson distribution reasonably accounts for the differences between the surface finishes seen in Fig. 2, it does not account for the differences in breakdown potential seen in Fig. 1 between $A O, C E$, and MP. This led to the supposition that if there is a distribution of flaws in the surface of the nitinol, what does the 
Table 2. Average breakdown potentials.

\begin{tabular}{|c|c|c|c|c|c|c|c|}
\hline & $0.05 \mathrm{~cm}^{2}$ & $0.1 \mathrm{~cm}^{2}$ & $0.5 \mathrm{~cm}^{2}$ & $1.0 \mathrm{~cm}^{2}$ & $2.0 \mathrm{~cm}^{2}$ & $5.0 \mathrm{~cm}^{2}$ & $10 \mathrm{~cm}^{2}$ \\
\hline w/ NB & 896 & 842 & 719 & 607 & 637 & 478 & 515 \\
\hline \multicolumn{8}{|c|}{ Mechanically polished } \\
\hline w/ NB & 863 & 934 & 875 & 669 & 737 & 560 & 350 \\
\hline w/ NB & 941 & 1000 & 875 & 776 & 898 & 452 & 455 \\
\hline w/o NB & 856 & - & 792 & 680 & 796 & 452 & 455 \\
\hline
\end{tabular}

Average breakdown potentials for AO, MP, and CE nitinol at different surface areas. "NB" stands for no breakdown.
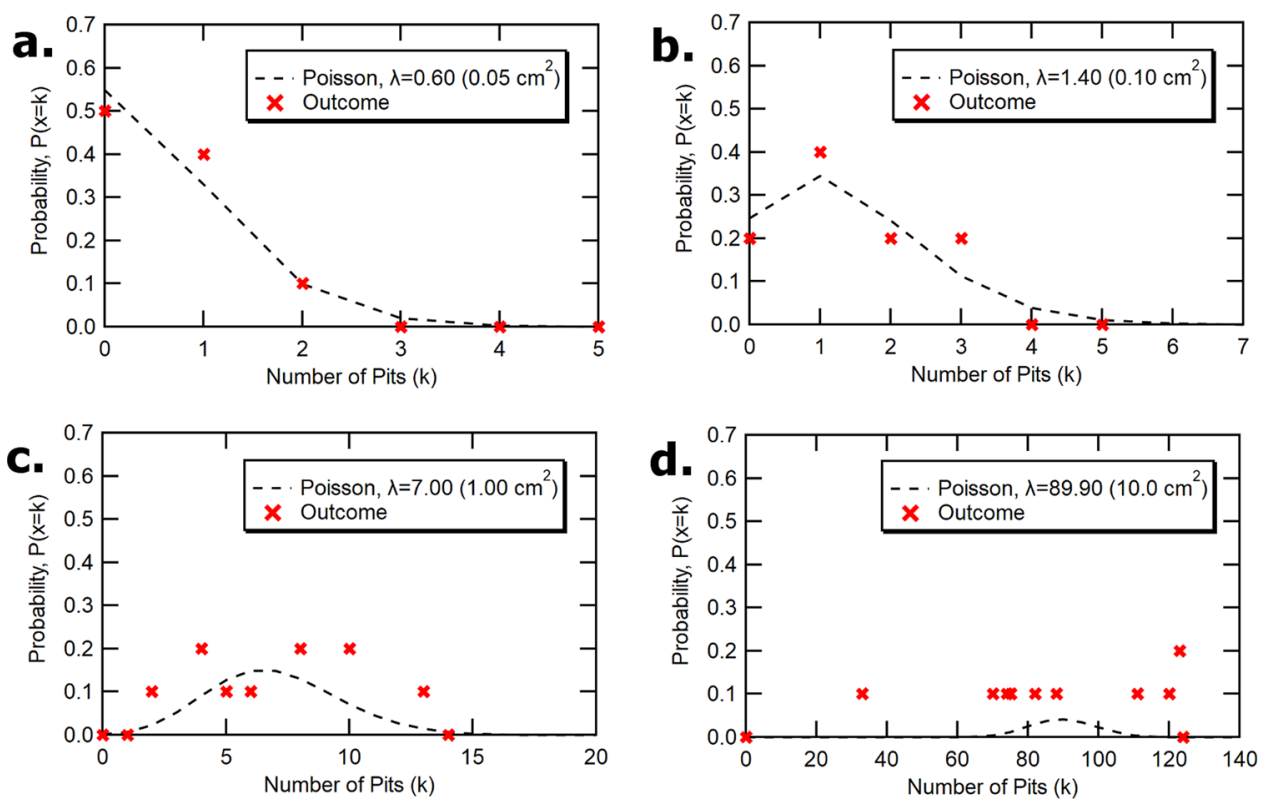

Fig. 4 Poisson distribution model of amber oxide samples. Poisson distribution of amber oxide samples at a 0.05 , b 0.10 , c 1.00 , and d $10.0 \mathrm{~cm}^{2}$.

distribution of the breakdown potentials for those flaws look like? This is shown in Fig. 3 where for each surface finish a bar graph of all breakdown potentials for all surface areas is shown. ASTM F2129 does not allow for the determination of the breakdown potential for each pit on a surface, instead it measures the breakdown potential of the flaw with the lowest breakdown potential on the exposed surface. Assuming the breakdown potential for the flaws is a normal distribution, it is expected that Fig. 3 would show either a normal distribution or a distribution skewed to lower breakdown potentials given the left censoring of ASTM F2129. Indeed, AO surface appeared to have a normal looking distribution, while CE and MP surface finishes had a flatter and broader distribution, and both seemed to have a median slightly lower than the AO surface finish. This raises an interesting question about the $A O$ surface finish as it appears in this study to have more flaws in the oxide layer, but those flaws seem to have a higher breakdown potential with less variability than the MP and CE surface finishes. While this would explain why the MP surface finish does not appear to plateau in Fig. 1 while the AO surface finish does, it does not explain why the CE surface finish converges when it has the same flaw distribution as the MP finish. Still, as ASTM F2129 measures the worst of the flaws, the number of flaws has a greater influence on the overall corrosion resistance than the median breakdown potential of the flaws. On a similar front, each finish had a threshold surface area above which electrochemical breakdown was observed for all specimens (with the exception of one specimen at $2.0 \mathrm{~cm}^{2} \mathrm{AO}$ ). For $\mathrm{AO}$, the threshold surface area was approximately $0.5 \mathrm{~cm}^{2}$, while for MP and $C E$, the threshold surface was approximately $5.0 \mathrm{~cm}^{2}$. Therefore, the minimum surface area required to assess the pitting corrosion susceptibility in a data set in which all specimens would be expected to experience an electrochemical breakdown increases with improved surface quality.

It is generally known that nitinol corrosion resistance depends highly on the surface finish and oxide layer ${ }^{15-17,27}$ and our results here confirm the importance of surface finish to breakdown potential. Although pitting corrosion can initiate from any number of surface flaws, inclusions have been the subject of recent investigations into pitting corrosion and may help explain why corrosion resistance decreased with increasing surface area in our study. One previous study that investigated the number of surface inclusions as nitinol was processed into tubes reported an increase in corrosion resistance with decreasing surface inclusion size ${ }^{28}$, which suggests that inclusions may be an important factor for the 

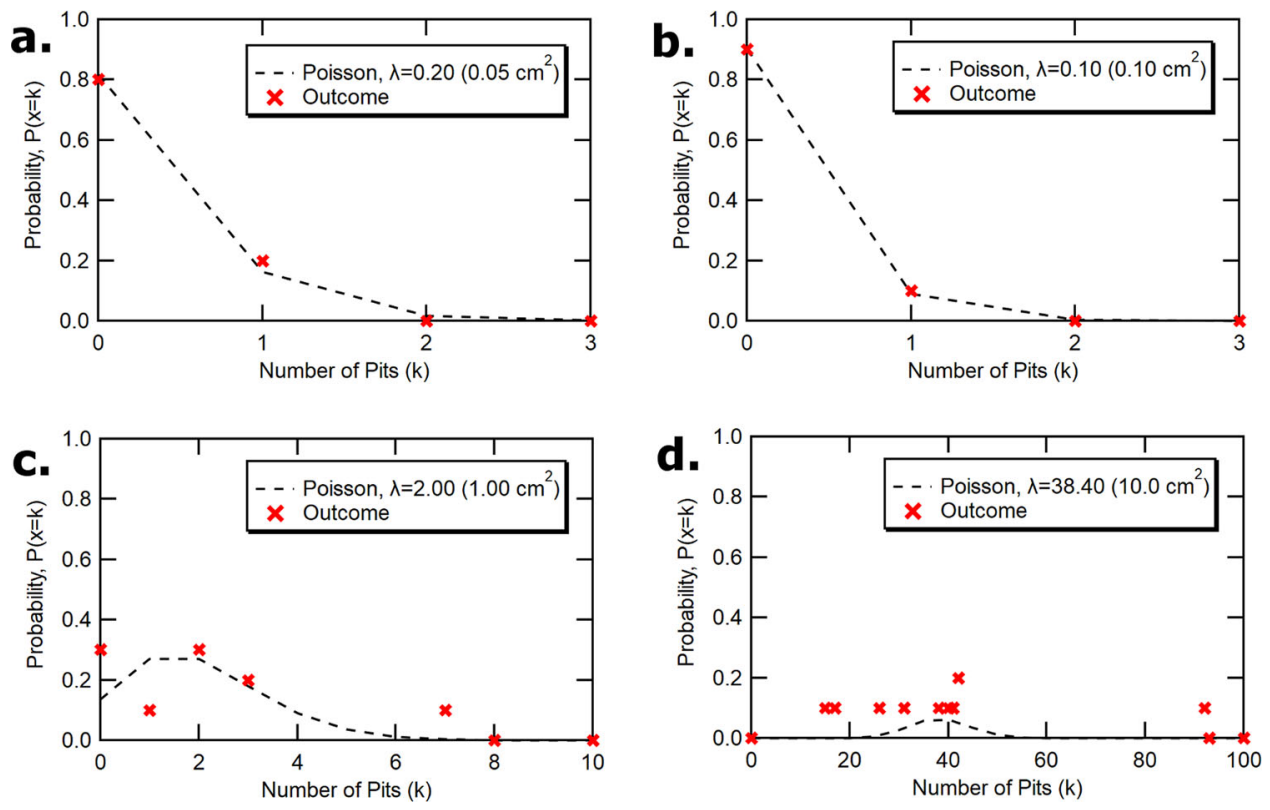

Fig. 5 Poisson distribution model of chemically etched samples. Poisson distribution of chemically etched samples at a 0.05 , b 0.10 , c 1.00 , and d $10.0 \mathrm{~cm}^{2}$.
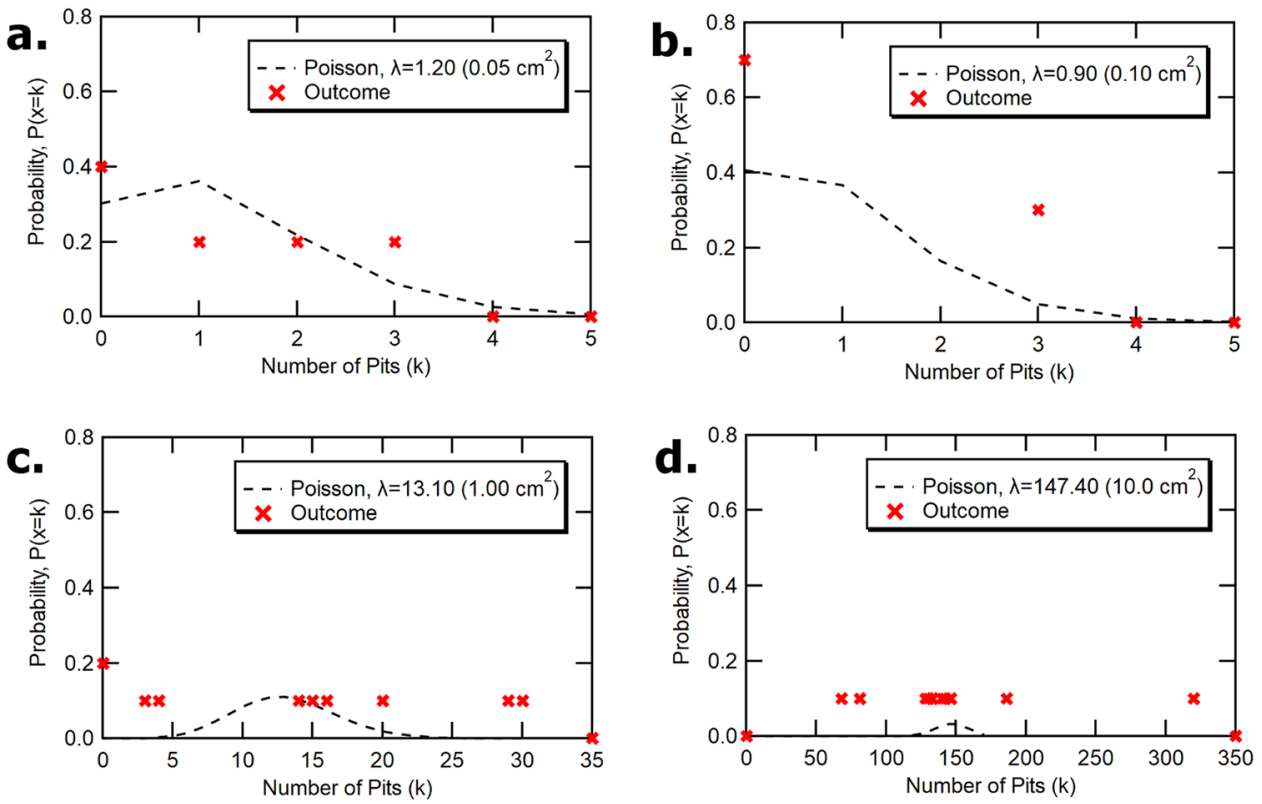

Fig. 6 Poisson distribution model of mechanically polished samples. Poisson distribution of mechanically polished samples at a 0.05 , b 0.10 , c 1.00 , and d $10.0 \mathrm{~cm}^{2}$.

initiation of pitting corrosion. Sun et al. ${ }^{29}$ examined corrosion susceptibility in nitinol stents and found an inverse relationship between inclusion fraction and breakdown potential. Another recent study furthermore suggests that pitting initiation in EP nitinol was associated with $\mathrm{Ti}_{2} \mathrm{NiO}_{x}$ inclusions and that titanium depletion in the surrounding alloy matrix may be the mechanism that renders the area more susceptible to pitting corrosion ${ }^{30}$. While it was not possible with our study design to analyze the dynamics of corrosion pit initiation nor was it within scope to conduct a systematic analysis of inclusions for each surface finish, it is logical that the differing surface finishes we tested would have varying distributions (size and area fraction) of surface inclusions that could have acted as corrosion pit initiation sites. As the surface area was decreased in our test specimens, the likelihood of each specimen containing a large inclusion (or other flaw) would have decreased as well resulting in higher recorded breakdown potentials as other (possibly smaller) flaws were able to initiate the first corrosion pit.

While some cardiovascular medical devices are made from wire, it is possible that wire and wire surface finishes would not be representative of the performance of non-wire based devices. While limited, the results from the laser-cut stents with a thermally grown oxide revealed that when a single stent ring was exposed during corrosion testing, half of the samples did not experience electrochemical breakdown, while in samples where 40 stent rings were exposed, all exhibited electrochemical breakdown. Although a limited sample size was used for our stent testing, the results seem to imply that the trends observed on wire specimens can be 


\begin{tabular}{|c|c|c|c|c|}
\hline $\begin{array}{l}\text { Surface } \\
\text { areas }\left(\mathrm{cm}^{2}\right)\end{array}$ & $\begin{array}{l}\text { Chi-square } \\
\left(X^{2}\right)\end{array}$ & $\begin{array}{l}\text { William's } \\
\text { correction }\end{array}$ & Critical value & Acceptable? \\
\hline \multicolumn{5}{|c|}{ a. Amber oxide } \\
\hline 0.05 & 0.195 & 0.172 & 6.635 & Yes \\
\hline 0.10 & 0.921 & 0.819 & 9.21 & Yes \\
\hline 0.50 & 9.206 & 8.123 & 11.345 & Yes \\
\hline 1.00 & 12.124 & 10.452 & 15.086 & Yes \\
\hline 2.00 & $6.22 \times 10^{2}$ & $5.29 \times 10^{2}$ & 16.812 & No \\
\hline 5.00 & $2.98 \times 10^{2}$ & $2.47 \times 10^{2}$ & 20.09 & No \\
\hline 10.0 & $3.22 \times 10^{10}$ & $2.70 \times 10^{10}$ & 18.475 & No \\
\hline \multicolumn{5}{|c|}{ b. Mechanically polished } \\
\hline 0.05 & 2.537 & 2.255 & 9.21 & Yes \\
\hline 0.10 & 20.137 & 17.899 & 9.21 & No \\
\hline 0.50 & $5.01 \times 10^{3}$ & $4.45 \times 10^{3}$ & 9.21 & No \\
\hline 1.00 & $2.01 \times 10^{5}$ & $1.69 \times 10^{5}$ & 18.475 & No \\
\hline 2.00 & $4.55 \times 10^{9}$ & $3.93 \times 10^{9}$ & 15.086 & No \\
\hline 5.00 & $1.69 \times 10^{8}$ & $1.42 \times 10^{8}$ & 18.475 & No \\
\hline 10.0 & $8.59 \times 10^{11}$ & $7.42 \times 10^{11}$ & 20.09 & No \\
\hline \multicolumn{5}{|c|}{ c. Chemically etched } \\
\hline 0.05 & 0.248 & 0.219 & 6.635 & Yes \\
\hline 0.10 & 0.056 & 0.049 & 6.635 & Yes \\
\hline 0.50 & 3.617 & 3.191 & 6.635 & Yes \\
\hline 1.00 & 30.261 & 26.701 & 11.345 & No \\
\hline 2.00 & 5.324 & 4.697 & 11.345 & Yes \\
\hline 5.00 & $1.77 \times 10^{7}$ & $1.49 \times 10^{7}$ & 18.475 & No \\
\hline 10.0 & $1.03 \times 10^{12}$ & $8.66 \times 10^{11}$ & 18.475 & No \\
\hline
\end{tabular}

applied to non-wire devices. These findings indicate that when it comes to medical device testing, for all surface finishes, the largest device will be the representative of worst-case condition for corrosion testing, while for some surface finishes intermediate sized devices could serve as a worst-case test condition. Thus, for all surface finishes, there was a significant effect of surface area on the pitting corrosion susceptibility indicating that even for surface finishes that do seem to converge to a breakdown potential at large surface area it may be important to ensure that smaller sized devices do not fall below the critical surface area. Overall, the data suggests that the largest device size should be considered as the worst-case for assessing pitting corrosion susceptibility when performing ASTM F2129.

In conclusion, this study has shown that surface area affects the breakdown potential based on ASTM F2129 with larger surface areas having lower breakdown potentials. The effect is modulated by the surface finish. For some nitinol surface finishes such as AO and $C E$, there was a surface area threshold above which the average breakdown potential plateaued. The observed trends were consistent across wires with different surface finishes as well as with laser cut stents. Poisson distribution model was a good fit for predicting the number of pits at smaller surface areas; however, more deviation was found at larger surface areas. The above conclusions support the need to consider the effect of surface area when selecting test specimens for pitting corrosion susceptibility testing.

\section{METHODS}

\section{Materials}

Four different surface finishes [AO, CE, MP, and EP] of Nitinol were selected. The nitinol wires all have a diameter of $0.5 \mathrm{~mm}$. Wires were tested as received. Nitinol wires were cut to length to achieve the desired surface area. For $A O, C E$, and MP surface finishes 10 samples were tested $(N=10)$. For EP surface finish six samples were tested $(N=6)$.

Commercially available laser-cut nitinol stents with a diameter of $7 \mathrm{~mm}$ and length of $100 \mathrm{~mm}$ were selected. Prior to corrosion susceptibility testing, the stents (which had a nominal EP finish) were subjected to air furnace heating at $540^{\circ} \mathrm{C}$ for about $2 \mathrm{~h}$ to thermally grow the oxide layer (Fig. 7a, b) to increase pitting corrosion susceptibility. A sample size of six $(N=6)$ was utilized for each test condition.

\section{Corrosion testing}

Wires were tested as per ASTM F2129 "Standard Test Method for Conducting Cyclic Potentiodynamic Polarization Measurements to Determine the Corrosion Susceptibility of Small Implant Devices" in deaerated PBS at a constant temperature of $37^{\circ} \mathrm{C}$. Prior to testing, PBS was deaerated for $30 \mathrm{~min}$ by sparging nitrogen gas at $150 \mathrm{~mL} / \mathrm{min}$. An Interface 1000 (Gamry, Warminster, PA) potentiostat, which was in compliance with ASTM G5 ${ }^{31}$ "Standard Reference Test Method for Making Potentiodynamic Anodic Polarization Measurements", was used to perform the corrosion test. Saturated calomel electrode (SCE) was used as reference electrode, while a graphite rod was used as counter electrode (Fig. 7e). The ASTM F2129 test started with an hour of open circuit potential to determine the rest potential, then followed by a cyclic polarization test that has a vertex potential of $1 \mathrm{~V}$ vs. SCE and back down to the rest potential at a scan rate of $1 \mathrm{mV} / \mathrm{s}$. Figure $7 \mathrm{f}$ shows representative curves of the cyclic potentiodynamic polarization test for each kind of specimen and Fig. 7e shows the three electrode test cell used for testing. Breakdown potential $\left(E_{\mathrm{b}}\right)$ was defined as shown in Fig. $7 f$ with a sudden increase in current.

The surface areas tested for $\mathrm{AO}, \mathrm{CE}$, and MP wires were $0.05,0.1,0.5,1.0$, $2.0,5.0$, and $10.0 \mathrm{~cm}^{2}$. For EP wires, the surface areas tested were 5.0 and $10.0 \mathrm{~cm}^{2}$. The samples were then attached to a sample holder with conductive silver paint (Fast Drying Silver Paint, Ted Pella, CA). The areas of attachment were coated twice with a non-conductive lacquer (Microstop, Tolber Chemical, AK) and dried overnight (Fig. 7c, d).

For the stent specimens, two different surface areas were tested. The smaller surface area of $0.34 \mathrm{~cm}^{2}$ was created by cutting the stent such that only one continuous ring of stent was exposed to the testing solution. The larger surface area of $13.5 \mathrm{~cm}^{2}$ had nearly 40 continuous rings of stent exposed. As with the wire samples, the stents were attached to a sample holder with conductive silver paint and twice coated with a nonconductive lacquer and dried overnight.

\section{Poisson distribution}

In addition to performing corrosion tests, the study also attempted to predict when corrosion will happen with a Poisson distribution model showed by Eq. (1).

$$
P(k)=e^{\wedge}(-\lambda) \lambda^{\wedge} k / k \text { ! }
$$

$P(k)$ models the probability of $k$ number events occurring in a given interval. Lambda $(\lambda)$ is the expected number of occurrences. This model will be applied by counting the number of pits in each surface area of the wire. In this case, $P(k)$ will be the probability that $k$ number of pits would occur per surface area, while $\lambda$ is the average number of pits per surface area. The model distribution will be then compared against the observed number of pits. This statistical approach has been used previously to establish a statistically significant sample size for corrosion testing ${ }^{20}$.

In order to count the number of pits, after the corrosion test, the wires were removed from the sample holder, rinsed with deionized water, and dried. The pits found in the entire length of the wire were counted under a stereo microscope (Nikon SMZ-10). It is expected that the pits will not appear uniformly, so for efficiency, clusters of pits were counted instead of individual ones (Fig. 7g). A cluster of pits (Fig. 7h) is considered as a group of multiple pits that appeared inside another pit.

\section{Statistical analysis}

Statistical analysis was performed using Minitab (Minitab Inc., State College, PA). The breakdown potentials, $E_{b}$, of all the samples at each 

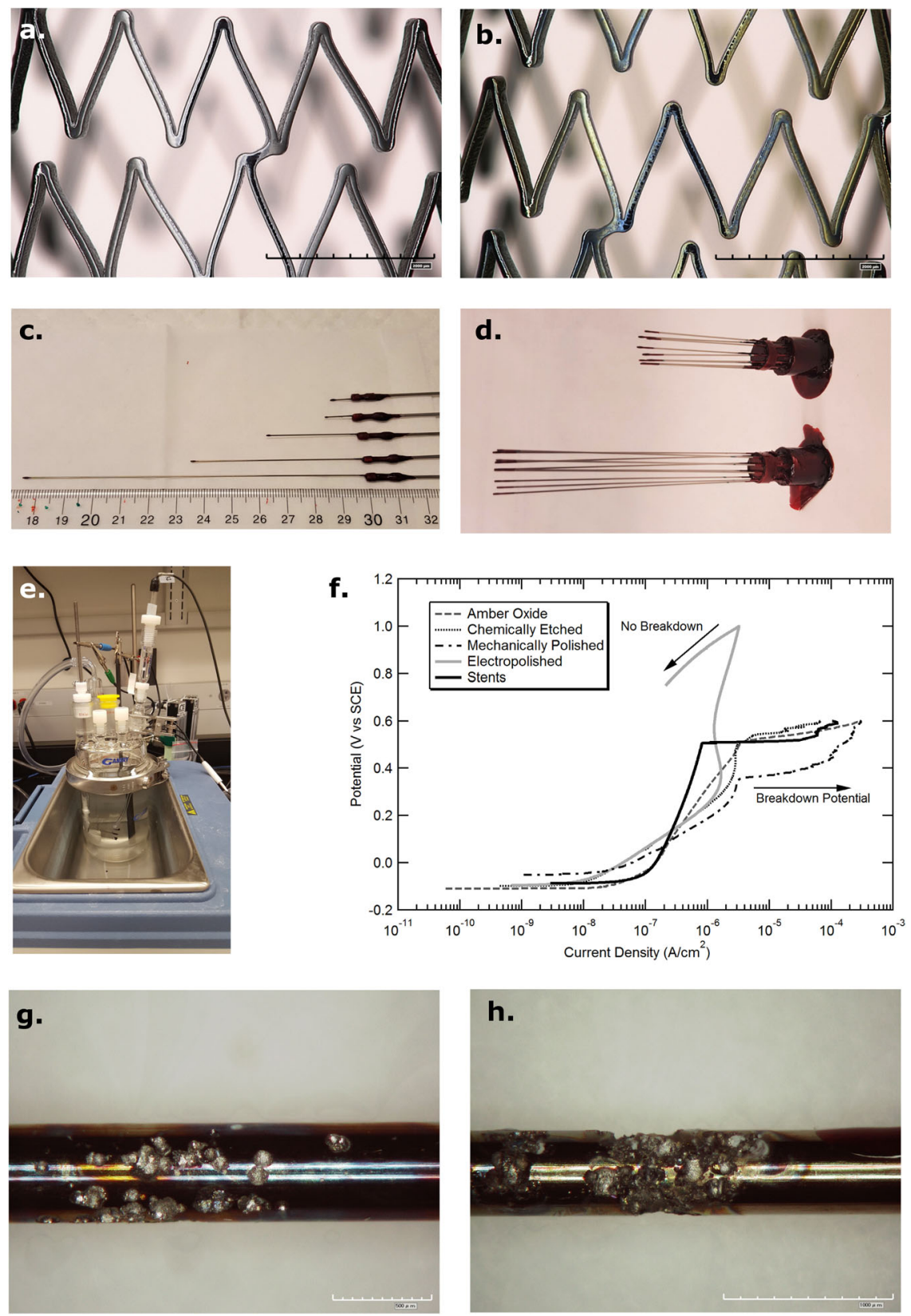

Fig. 7 Sample preparation and test set up. Optical microscopy image of stents $\mathbf{a}$ before and $\mathbf{b}$ after subjecting to air furnace heating to thermally grow an oxide layer. Sample preparation for $\mathbf{c} 0.05-2.00 \mathrm{~cm}^{2}$ and $\mathbf{d} 5.00-10.0 \mathrm{~cm}^{2}$ after they were coated with a conductive silver paint and nonconductive lacquer. $f$ Representative curves of the cyclic potentiodynamic polarization tests for each kind of specimen performed on e a three-electrode cell. Digital microscopy images of post-corrosion amber oxide samples with $\mathbf{g}$ individual and $\mathbf{h}$ cluster of pits.

surface area were compared through a Log Rank test with right censoring. This is because when the samples did not experience a breakdown, $E_{\mathrm{b}}$ was assumed to be $1000 \mathrm{mV}$. Statistical significance was set at $a<0.05$. Additionally, a chi-square $\left(X^{2}\right)$ test performed in MS Excel (Microsoft Corp., Redmond, WA) was used to determine the goodnessof-fit between the Poisson distribution model and the observed number of pits. Since the sample size is small, William's correction factor was used.

\section{DATA AVAILABILITY}

The data that support the findings of this study are available from the corresponding author upon reasonable request.
Received: 3 April 2020; Accepted: 17 July 2020;

Published online: 06 August 2020

\section{REFERENCES}

1. Duerig, T., Pelton, A. \& Stöckel, D. An overview of nitinol medical applications Mater. Sci. Eng. A 273, 149-160 (1999).

2. Pelton, A., Duerig, T. \& Stöckel, D. A guide to shape memory and superelasticity in Nitinol medical devices. Minim. Invasive Ther. 13, 218-221 (2004).

3. Kolotyrkin, J. M. Pitting corrosion of metals. Corrosion 19, 261t-268t (1963).

4. Stoeckel, D., Pelton, A. \& Duerig, T. Self-expanding nitinol stents: material and design considerations. Eur. Radiol. 14, 292-301 (2004). 
5. Warner, C. P. The effect of exposure to simulated body fluids on breakdown potentials. J. Mater. Eng. Perform. 18, 754-759 (2009).

6. ASTM International. ASTM F2129-17, Standard Test Method for Conducting Cyclic Potentiodynamic Polarization Measurements to Determine the Corrosion Susceptibility of Small Implant Devices. (ASTM International, West Conshohocken, PA, 2017).

7. Jones, D. A. Principles and Prevention of Corrosion (Macmillan, 1992).

8. Corbett, R. A. Laboratory Corrosion Testing of Medical Implants, In Materials and Processes for Medical Devices Conference, Anaheim, California, September 8-10, 2003. Proceedings. (ed. Shrivastava, S.) 166-171 (ASM International, 2004).

9. Thyssen, J. P., Linneberg, A., Menné, T. \& Johansen, J. D. The epidemiology of contact allergy in the general population-prevalence and main findings. Contact Derm. 57, 287-299 (2007).

10. Rahimipour, S., Salahinejad, E., Sharifi, E., Nosrati, H. \& Tayebi, L. Structure, wettability, corrosion and biocompatibility of nitinol treated by alkaline hydrothermal and hydrophobic functionalization for cardiovascular applications. Appl. Surf. Sci. 506, 144657 (2020).

11. Ryhänen, J. et al. Biocompatibility of nickel-titanium shape memory metal and its corrosion behavior in human cell cultures. JBMRGL 35, 451-457 (1997).

12. U.S. Food and Drug Administration. Guidance for Industry and Food and Drug Administration Staff: Use of International Standard ISO 10993-1, "Biological evaluation of medical devices - Part 1: Evaluation and testing within a risk management process." (2016).

13. International Organization for Standardization. ISO 10993-1, Biological evaluation of medical devices - Part 1: Evaluation and testing within a risk management process. (2018).

14. Sivan, S., Di Prima, M. \& Weaver, J. D. Effect of applied potential on fatigue life of electropolished nitinol wires. SMST 3, 238-249 (2017)

15. Trepanier, C., Tabrizian, M., Yahia, L. H., Bilodeau, L. \& Piron, D. L. Effect of modification of oxide layer on NiTi stent corrosion resistance. J. Biomed. Mater. Res. 43, 433-440 (1998).

16. Pound, B. G. Corrosion behavior of metallic materials in biomedical applications. I. Ti and its alloys. Corros. Rev. 32, 1-20 (2014).

17. Sullivan, S. J. L. et al. Effects of oxide layer composition and radial compression on nickel release in nitinol stents. SMST 1, 319-327 (2015).

18. Nissan, A., Eiselstein, L., Steffey, D. \& Corlett, N. Effect of long-term immersion on the pitting corrosion resistance of nitinol. in International Conference on Shape Memory and Superelastic Technologies, SMST-2007, Tsukuba, Japan, December 3-5, 2007. Proceedings. (eds. Berg, B., Mitchell, M.R. \& Proft, J.) 271-278 (ASM International, 2008).

19. Di Prima, M. A., Guiterrez, E. \& Weaver, J. The effect of fatigue on the corrosion resistance of common medical alloys. J. Biomed. Mater. Res. Part B Appl. Biomater. 105, 2019-2026 (2017).

20. Eiselstein, L. E. et al. Acceptance criteria for corrosion resistance of medical devices: statistical analysis of nitinol pitting in in vivo environments. J. Mater. Eng. Perform. 18, 768-780 (2009).

21. Siddiqui, D. A., Sivan, S., Weaver, J. D. \& Di Prima, M. Effect of wire fretting on the corrosion resistance of common medical alloys. J. Biomed. Mater. Res. Part B Appl. Biomater. 105, 2487-2494 (2016)

22. Lonn, M. K., Metcalf, J. M. \& Choules, B. D. In vivo and in vitro nitinol corrosion properties. SMST 1, 328-338 (2015)

23. Pound, B. G. The electrochemical behavior of nitinol in simulated gastric fluid. $J$. Biomed. Mater. Res. Part B Appl. Biomater. 105, 2394-2400 (2017).

24. Pound, B. G. Corrosion behavior of nitinol in blood serum and PBS containing amino acids. J. Biomed. Mater. Res. Part B Appl. Biomater. 94, 287-295 (2010).

25. Zende, S., Freiberg, K. E., Dorner, F., Feth, N.-A. \& Undisz, A. Corrosion Resistance of Nitinol Wires After Deformation. SMST 5, 346-351 (2019).

26. Nagaraja, S., Di Prima, M., Saylor, D. \& Takai, E. Current practices in corrosion, surface characterization, and nickel leach testing of cardiovascular metallic implants. J. Biomed. Mater. Res. Part B Appl. Biomater. 105, 1330-1341 (2017).

27. Zhu, L., Fino, J. \& Pelton, A. Oxidation of Nitinol. in International Conference on Shape Memory and Superelastic Technologies, SMST-2003, Pacific Grove, California, May 5-8, 2003. Proceedings. (eds. Pelton, A. \& Duerig, T.) 367-373 (SMST Society, Inc., 2004).

28. Wohlschlögel, M., Steegmüller, R. \& Schüßler, A. Effect of inclusion size and distribution on the corrosion behavior of medical-device grade nitinol tubing. J. Mater. Eng. Perform. 23, 2635-2640 (2014).
29. Sun, F. et al. On the high sensitivity of corrosion resistance of NiTi stents with respect to inclusions: an experimental evidence. ACS Omega 5, 3073-3079 (2020).

30. Pound, B. G. Pit initiation on nitinol in simulated physiological solutions. J. Biomed. Mater. Res. Part B Appl. Biomater. 106, 1605-1610 (2018).

31. ASTM International. ASTM G5-14, Standard reference test method for making potentiostatic and potentiodynamic anodic polarization measurements. (ASTM International, West Conschohocken, PA, 2014).

\section{ACKNOWLEDGEMENTS}

This project was funded by the Division of Applied Mechanics. Similarly, it was supported in part by an appointment to the Research Participation Program at the Center for Devices and Radiological Health administered by the Oak Ridge Institute for Science and Education through an interagency agreement between the U.S. Department of Energy and the U.S. Food and Drug Administration. The mention of commercial products, their sources, or their use in connection with material reported herein is not to be construed as either an actual or implied endorsement of such products by the Department of Health and Human Services. The findings and conclusions in this article have not been formally disseminated by the Food and Drug Administration and should not be construed to represent any Agency determination or policy.

\section{AUTHOR CONTRIBUTIONS}

All authors have made substantial contributions to the conception of the work, acquisition, analysis and interpretation of data. All authors also approved the submitted version and agreed to be both personally accountable for the author's own contributions and to ensure that questions related to the accuracy or integrity of any part of the work, even ones in which the author was not personally involved, are appropriately investigated, resolved, and the resolution documented in the literature.

\section{COMPETING INTERESTS}

The authors declare no competing interests.

\section{ADDITIONAL INFORMATION}

Supplementary information is available for this paper at https://doi.org/10.1038/ s41529-020-00128-3.

Correspondence and requests for materials should be addressed to M.D.P.

Reprints and permission information is available at http://www.nature.com/ reprints

Publisher's note Springer Nature remains neutral with regard to jurisdictional claims in published maps and institutional affiliations.

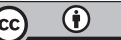

Open Access This article is licensed under a Creative Commons Attribution 4.0 International License, which permits use, sharing, adaptation, distribution and reproduction in any medium or format, as long as you give appropriate credit to the original author(s) and the source, provide a link to the Creative Commons license, and indicate if changes were made. The images or other third party material in this article are included in the article's Creative Commons license, unless indicated otherwise in a credit line to the material. If material is not included in the article's Creative Commons license and your intended use is not permitted by statutory regulation or exceeds the permitted use, you will need to obtain permission directly from the copyright holder. To view a copy of this license, visit http://creativecommons. org/licenses/by/4.0/.

This is a U.S. government work and not under copyright protection in the U.S.; foreign copyright protection may apply 2020, corrected publication 2021 